CERN-PPE/94-22

4 February 1994

\title{
A measurement of the $B_{s}^{0} \quad$ sw s 409 meson mass
}

DELPHI Collaboration

\begin{abstract}
Strange beauty mesons $\mathrm{B}_{s}^{0}$ have been reconstructed through exclusive hadronic final states, using the data collected in 1992 by the DELPHI experiment at the LEP collider. The analysis relies on the combined use of accurate tracking and of the hadron identification capabilities of DELPHI. Three $\mathrm{B}_{s}^{0}$ decay candidates have been selected over a negligible background using the $\mathrm{D}_{s}^{-} \pi^{+}$, $\mathrm{D}_{s}^{-} \mathrm{a}_{1}^{+}(1260)$ and $\mathrm{J} / \psi \phi$ decay modes, with identified kaons in the final state. The $\mathrm{B}_{s}^{0}$ meson mass is measured to be $\mathrm{m}_{\mathrm{B}_{\mathrm{s}}}=(5374 \pm 16 \pm 2) \mathrm{MeV} / \mathrm{c}^{2}$.
\end{abstract}

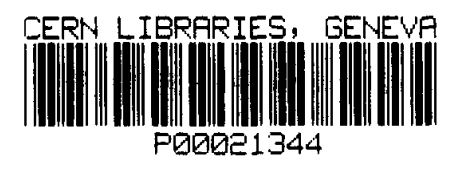

(Submitted to Physics Letters B) 
P.Abreu ${ }^{20}$, W.Adam ${ }^{7}$, T.Adye ${ }^{37}$, E.Agasi ${ }^{30}$, R.Aleksan ${ }^{39}$, G.D.Alekseev ${ }^{14}$, P.Allport ${ }^{21}$, S.Almehed ${ }^{23}$, F.M.L.Almeida Junior ${ }^{47}, \quad$ S.J.Alvsvaag ${ }^{4}, \quad$ U.Amaldi $^{7}, \quad$ A.Andreazza $^{27}, \quad$ P.Antilogus ${ }^{24}, \quad$ W-D.Apel ${ }^{15}$, R.J.Apsimon ${ }^{37}$, Y.Amoud ${ }^{39}$, B.Asman ${ }^{44}$, J-E.Augustin ${ }^{18}$, A.Augustinus ${ }^{30}$, P.Baillon ${ }^{7}$, P.Bambade ${ }^{18}$, F.Barao $^{20}$, R.Barate ${ }^{12}$, G.Barbiellini ${ }^{46}$, D.Y.Bardin ${ }^{14}$, G.J.Barker ${ }^{34}$, A.Baroncelli ${ }^{40}$, O.Barring ${ }^{7}$, J.A.Barrio ${ }^{25}$, W.Bartl $^{50}$, M.J.Bates ${ }^{37}$, M.Battaglia ${ }^{13}$, M.Baubillier ${ }^{22}$, K-H.Becks ${ }^{52}$, M.Begalli ${ }^{36}$, P.Beilliere ${ }^{6}$, Yu.Belokopytov ${ }^{42}$, P.Beltran ${ }^{9}$, A.C.Benvenuti ${ }^{5}, \quad$ M.Berggren ${ }^{18}$, D.Bertrand ${ }^{2}$, F.Bianchi ${ }^{45}$, M.Bigi $^{45}$, M.S.Bilenky ${ }^{14}$, P.Billoir ${ }^{22}$, J.Bjarne ${ }^{23}$, D.Bloch ${ }^{8}$, J.Blocki ${ }^{51}$, S.Blyth ${ }^{34}$, V.Bocci ${ }^{38}$, P.N.Bogolubov ${ }^{14}$, T.Bolognese ${ }^{39}$, M.Bonesini ${ }^{27}$, W.Bonivento ${ }^{27}$, P.S.L.Booth ${ }^{21}$, G.Borisov ${ }^{42}$, C.Bosio ${ }^{40}$, B.Bostjancic ${ }^{43}$, S.Bosworth $^{34}$, O.Botner ${ }^{48}$, B.Bouquet ${ }^{18}$, C.Bourdarios ${ }^{18}$, T.J.V.Bowcock ${ }^{21}$, M.Bozzo ${ }^{11}$, S.Braibant ${ }^{2}$, P.Branchini $^{40}$, K.D.Brand ${ }^{35}$, R.A.Brenner ${ }^{13}$, H.Briand ${ }^{22}$, C.Bricman ${ }^{2}$, L.Brillault ${ }^{22}$, R.C.A.Brown ${ }^{7}$, P.Bruckman $^{16}$, J-M.Brunet ${ }^{6}$, A.Budziak ${ }^{16}$, L.Bugge ${ }^{32}$, T.Buran ${ }^{32}$, A.Buys ${ }^{7}$, J.A.M.A.Buytaert ${ }^{7}$, M.Caccia ${ }^{27}$, M.Calvi ${ }^{27}$, A.J.Camacho Rozas ${ }^{41}$, R.Campion ${ }^{21}$, T.Camporesi ${ }^{7}$, V.Canale ${ }^{38}$, K.Cankocak ${ }^{44}$, F.Cao $^{2}$, F.Carena ${ }^{7}$, P.Carrilho $^{47}$, L.Carroll ${ }^{21}$, R.Cases ${ }^{49}$, C.Caso ${ }^{11}$, M.V.Castillo Gimenez ${ }^{49}$, A.Cattai ${ }^{7}$, F.R.Cavallo ${ }^{5}$, L.Cerrito ${ }^{38}$, V.Chabaud ${ }^{7}$, A.Chan ${ }^{1}$, M.Chapkin ${ }^{42}$, Ph.Charpentier ${ }^{7}$, J.Chauveau ${ }^{22}$, P.Checchia ${ }^{35}$, G.A.Chelkov ${ }^{14}$, L.Chevalier $^{39}$, P.Chliapnikov ${ }^{42}$, V.Chorowicz ${ }^{22}$, J.T.M.Chrin ${ }^{49}$, V.Cindro ${ }^{43}$, P.Collins ${ }^{34}$, J.L.Contreras ${ }^{18}$, R.Contri ${ }^{11}$, E.Cortina ${ }^{49}$, G.Cosme ${ }^{18}$, F.Couchot ${ }^{18}$, H.B.Crawley ${ }^{1}$, D.Crennell ${ }^{37}$, G.Crosetti ${ }^{11}$, J.Cuevas Maestro ${ }^{33}$, S.Czellar ${ }^{13}$, E.Dahl-Jensen ${ }^{28}$, J.Dahm ${ }^{52}$, B.Dalmagne ${ }^{18}$, M.Dam ${ }^{32}$, G.Damgaard ${ }^{28}$, E.Daubie $^{2}$, A.Daum ${ }^{15}$, P.D.Dauncey ${ }^{7}$, M.Davenport ${ }^{7}$, J.Davies ${ }^{21}$, W.Da Silva ${ }^{22}$, C.Defoix ${ }^{6}$, P.Delpierre ${ }^{26}$, N.Demaria ${ }^{34}$ A.De Angelis ${ }^{7}$, H.De Boeck ${ }^{2}$, W.De Boer ${ }^{15}$, S.De Brabandere ${ }^{2}$, C.De Clercq ${ }^{2}$, M.D.M.De Fez Laso ${ }^{49}$, C.De La Vaissiere ${ }^{22}$, B.De Lotto ${ }^{46}, \quad$ A.De Min ${ }^{27}$, L.De Paula ${ }^{47}$, H.Dijkstra ${ }^{7}$, L.Di Ciaccio ${ }^{38}$, F.Djama ${ }^{8}$, J.Dolbeau ${ }^{6}$, M.Donszelmann ${ }^{7}$, K.Doroba ${ }^{51}$, M.Dracos ${ }^{8}$, J.Drees ${ }^{52}$, M.Dris ${ }^{31}$, Y.Dufour $^{7}$, F.Dupont ${ }^{12}$, D.Edsall ${ }^{1}$, L-O.Eek ${ }^{48}$, R.Ehret ${ }^{15}$, T.Ekelof ${ }^{48}$, G.Ekspong ${ }^{44}$, A.Elliot Peisert ${ }^{7}$, M.Elsing ${ }^{52}$, J-P.Engel ${ }^{8}$, N.Ershaidat ${ }^{22}$, M.Espirito Santo ${ }^{20}$, D.Fassouliotis ${ }^{31}$, M.Feindt ${ }^{7}$, A.Fenyuk $^{42}$, A.Ferrer $^{49}$, T.A.Filippas ${ }^{31}$, A.Firestone ${ }^{1}$, H.Foeth ${ }^{7}$, E.Fokitis ${ }^{31}$, F.Fontanelli ${ }^{11}$, K.A.J.Forbes ${ }^{21}$, F.Formenti ${ }^{7}$, J-L.Fousset $^{26}$, S.Francon ${ }^{24}$, B.Franek ${ }^{37}$, P.Frenkiel ${ }^{6}$, D.C.Fries ${ }^{15}$, A.G.Frodesen ${ }^{4}, \quad$ R.Fruhwirth $^{50}$, F.Fulda-Quenzer ${ }^{18}$, H.Furstenau ${ }^{7}$, J.Fuster ${ }^{7}$, D.Gamba ${ }^{45}$, M.Gandelman ${ }^{17}$, C.Garcia ${ }^{49}$, J.Garcia ${ }^{41}$, C.Gaspar $^{7}$, U.Gasparini $^{35}$, Ph.Gavillet ${ }^{7}$, E.N.Gazis ${ }^{31}$, J-P.Gerber ${ }^{8}$, P.Giacomelli ${ }^{7}$, D.Gillespie ${ }^{7}$, R.Gokieli ${ }^{51}$, B.Golob ${ }^{43}$, V.M.Golovatyuk ${ }^{14}$, J.J.Gomez Y Cadenas ${ }^{7}$, G.Gopal ${ }^{37}$, L.Gorn ${ }^{1}$, M.Gorski ${ }^{51}$, V.Gracco ${ }^{11}$, F.Grard ${ }^{2}$, E.Graziani ${ }^{40}$, G.Grosdidier ${ }^{18}$, B.Grossetete ${ }^{22}$, P.Gunnarsson ${ }^{44}$, J.Guy ${ }^{37}$, U.Haedinger ${ }^{15}$, F.Hahn ${ }^{52}$, M.Hahn ${ }^{44}$, S.Hahn $^{52}$, S.Haider ${ }^{30}$, Z.Hajduk ${ }^{16}$, A.Hakansson ${ }^{23}$, A.Hallgren ${ }^{48}$, K.Hamacher ${ }^{52}$, G.Hamel De Monchenault ${ }^{39}$, W.Hao $^{30}$, F.J.Harris ${ }^{34}$, V.Hedberg ${ }^{23}$, R.Henriques ${ }^{20}$, J.J.Hernandez ${ }^{49}$, J.A.Hernando ${ }^{49}$, P.Herquet ${ }^{2}$, H.Herr ${ }^{7}$,

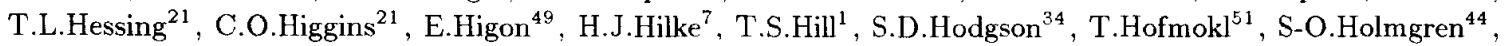
P.J.Holt ${ }^{34}$, D.Holthuizen ${ }^{30}$, P.F.Honore ${ }^{6}$, M.Houlden ${ }^{21}$, J.Hrubec ${ }^{50}$, K.Huet ${ }^{2}$, K.Hultquist ${ }^{44}$, P.Ioannou ${ }^{3}$, P-S.Iversen ${ }^{4}$, J.N.Jackson ${ }^{21}$, R.Jacobsson ${ }^{44}$, P.Jalocha ${ }^{16}, \quad$ G.Jarlskog ${ }^{23}$, P.Jarry ${ }^{39}$, B.Jean-Marie ${ }^{18}$, E.K.Johansson ${ }^{44}$, M.Jonker ${ }^{7}$, L.Jonsson ${ }^{23}$, P.Juillot ${ }^{8}$, M.Kaiser ${ }^{15}$, G.Kalkanis ${ }^{3}$, G.Kalmus ${ }^{37}$, F.Kapusta ${ }^{22}$, M.Karlsson ${ }^{44}$, E.Karvelas ${ }^{9}$, S.Katsanevas ${ }^{3}$, E.C.Katsoufis ${ }^{31}$, R.Keranen ${ }^{7}$, B.A.Khomenko ${ }^{14}$, N.N.Khovanski ${ }^{14}$, B.King ${ }^{21}$, N.J.Kjaer ${ }^{28}$, H.Klein ${ }^{7}$, A.Klovning ${ }^{4}$, P.Kluit ${ }^{30}$, A.Koch-Mehrin ${ }^{52}$, J.H.Koehne ${ }^{15}$, B.Koene ${ }^{30}$, P.Kokkinias $^{9}$, M.Koratzinos ${ }^{32}$, K.Korcyl ${ }^{16}$, A.V.Korytov ${ }^{14}$, V.Kostioukhine ${ }^{42}$, C.Kourkoumelis ${ }^{3}$, O.Kouznetsov ${ }^{14}$, P.H.Kramer ${ }^{52}$, C.Kreuter ${ }^{15}$, J.Krolikowski ${ }^{51}$, I.Kronkvist ${ }^{23}$, W.Krupinski ${ }^{16}$, W.Kucewicz ${ }^{16}$, K.Kulka ${ }^{48}$, K.Kurvinen ${ }^{13}$, C.Lacasta ${ }^{49}$, C.Lambropoulos ${ }^{9}$, J.W.Lamsa ${ }^{1}$, L.Lanceri ${ }^{46}$, P.Langefeld ${ }^{52}$, V.Lapin ${ }^{42}$, I.Last $^{21}$, J-P.Laugier ${ }^{39}$, R.Lauhakangas ${ }^{13}$, G.Leder ${ }^{50}$, F.Ledroit ${ }^{12}$, R.Leitner ${ }^{29}$, Y.Lemoigne ${ }^{39}$, J.Lemonne ${ }^{2}$,

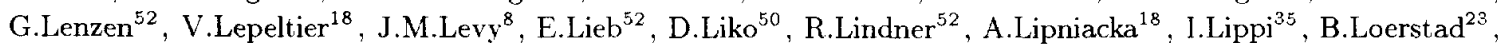
M.Lokajicek ${ }^{10}$, J.G.Loken ${ }^{34}$, A.Lopez-Fernandez ${ }^{7}$, M.A.Lopez Aguera ${ }^{41}$, M.Los ${ }^{30}$, D.Loukas ${ }^{9}$, J.J.Lozano ${ }^{49}$,

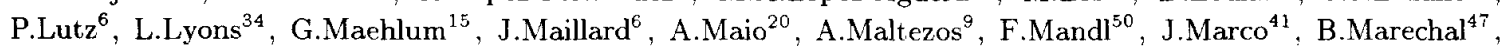
M.Margoni $^{35}$, J-C.Marin ${ }^{7}$, C.Mariotti ${ }^{40}$, A.Markou ${ }^{9}$, T.Maron ${ }^{52}$, S.Marti ${ }^{49}$, C.Martinez-Rivero ${ }^{41}$, F.Martinez-Vidal ${ }^{49}$, F.Matorras ${ }^{41}$, C.Matteuzzi ${ }^{27}$, G.Matthiae ${ }^{38}$, M.Mazzucato ${ }^{35}$, M.Mc Cubbin ${ }^{21}$, R.Mc Kay ${ }^{1}$, R.Mc Nulty $^{21}$, J.Medbo ${ }^{48}$, C.Meroni ${ }^{27}$, W.T.Meyer ${ }^{1}$, M.Michelotto ${ }^{35}$, E.Migliore ${ }^{45}$, I.Mikulec ${ }^{50}$, L.Mirabito ${ }^{24}$, W.A.Mitarof ${ }^{50}$, G.V.Mitselmakher ${ }^{14}$, U.Mjoernmark ${ }^{23}$, T.Moa ${ }^{44}$, R.Moeller ${ }^{28}$, K.Moenig ${ }^{7}$, M.R.Monge ${ }^{11}$, P.Morettini $^{11}$, H.Mueller ${ }^{15}$, W.J.Murray ${ }^{37}$, B.Muryn ${ }^{16}$, G.Myatt ${ }^{34}$, F.Naraghi ${ }^{12}$, F.L.Navarria ${ }^{5}$, P.Negri ${ }^{27}$, S.Nemecek $^{10}$, W.Neumann ${ }^{52}$, N.Neumeister ${ }^{50}$, R.Nicolaidou ${ }^{3}$, B.S.Nielsen ${ }^{28}$, V.Nikolaenko ${ }^{42}$, P.E.S.Nilsen ${ }^{4}$, P.Niss $^{44}$ A.Nomerotski ${ }^{35}$, M.Novak ${ }^{10}$, V.Obraztsov ${ }^{42}$, A.G.Olshevski ${ }^{14}$, R.Orava ${ }^{13}$, A.Ostankov ${ }^{42}$, K.Osterberg $^{13}$, A.Ouraou ${ }^{39}$, P.Paganini ${ }^{18}$, M.Paganoni ${ }^{27}$, R.Pain ${ }^{22}$, H.Palka ${ }^{16}$, Th.D.Papadopoulou ${ }^{31}$, L.Pape ${ }^{7}$, F.Parodi ${ }^{11}$, A.Passeri ${ }^{40}$, M.Pegoraro ${ }^{35}$, J.Pennanen ${ }^{13}$, L.Peralta ${ }^{20}$, H.Pernegger ${ }^{50}$, M.Pernicka ${ }^{50}$, A.Perrotta ${ }^{5}$, C.Petridou $^{46}$, A.Petrolini ${ }^{11}$, G.Piana ${ }^{11}$, F.Pierre ${ }^{39}$, M.Pimenta ${ }^{20}$, S.Plaszczynski ${ }^{18}$, O.Podobrin ${ }^{15}$, M.E.Pol ${ }^{17}$, G.Polok $^{16}$, P.Poropat ${ }^{46}$, V.Pozdniakov ${ }^{14}$, M.Prest ${ }^{46}$, P.Privitera ${ }^{38}$, A.Pullia ${ }^{27}$, D.Radojicic ${ }^{34}$, S.Ragazzi ${ }^{27}$, H.Rahmani ${ }^{31}$, P.N.Ratoff ${ }^{19}$, A.L.Read ${ }^{32}$, M.Reale ${ }^{52}$, P.Rebecchi ${ }^{18}$, N.G.Redaelli ${ }^{27}$, M.Regler ${ }^{50}$, D.Reid ${ }^{7}$, P.B.Renton ${ }^{34}$, L.K.Resvanis ${ }^{3}$, F.Richard ${ }^{18}$, J.Richardson ${ }^{21}$, J.Ridky ${ }^{10}$, G.Rinaudo ${ }^{45}$, A.Romero ${ }^{45}$, I.Roncagliolo $^{11}$, P.Ronchese ${ }^{35}$, E.I.Rosenberg ${ }^{1}$, E.Rosso ${ }^{7}$, P.Roudeau ${ }^{18}$, T.Rovelli ${ }^{5}$, W.Ruckstuhl ${ }^{30}$, 
V.Ruhlmann-Kleider ${ }^{39}$, A.Ruiz ${ }^{41}$, H.Saarikko ${ }^{13}$, Y.Sacquin ${ }^{39}$, G.Sajot ${ }^{12}$, J.Salt ${ }^{49}$, J.Sanchez ${ }^{25}$, M.Sannino ${ }^{11}$, S.Schael $^{7}$, H.Schneider ${ }^{15}$, M.A.E.Schyns ${ }^{52}$, G.Sciolla ${ }^{45}$, F.Scuri ${ }^{46}$, A.M.Segar ${ }^{34}$, A.Seitz ${ }^{15}$, R.Sekulin ${ }^{37}$, M.Sessa $^{46}$, R.Seufert ${ }^{15}$, R.C.Shellard ${ }^{36}$, I.Siccama ${ }^{30}$, P.Siegrist ${ }^{39}$, S.Simonetti ${ }^{11}$, F.Simonetto ${ }^{35}$, A.N.Sisakian ${ }^{14}$, G.Skjevling ${ }^{32}$, G.Smadja ${ }^{39,24}$, N.Smirnov ${ }^{42}$, O.Smirnova ${ }^{14}$, G.R.Smith ${ }^{37}$, R.Sosnowski ${ }^{51}$, D.Souza-Santos ${ }^{36}$, T.Spassov ${ }^{20}$, E.Spiriti ${ }^{40}$, S.Squarcia ${ }^{11}$, H.Staeck ${ }^{52}$, C.Stanescu ${ }^{40}$, S.Stapnes ${ }^{32}$, G.Stavropoulos ${ }^{9}$, F.Stichelbaut ${ }^{7}$, A.Stocchi ${ }^{18}$, J.Strauss ${ }^{50}$, J.Straver ${ }^{7}$, R.Strub ${ }^{8}$, B.Stugu ${ }^{4}$, M.Szczekowski ${ }^{51}$, M.Szeptycka ${ }^{51}$, P.Szymanski ${ }^{51}$, T.Tabarelli $^{27}$, O.Tchikilev ${ }^{42}$, G.E.Theodosiou ${ }^{9}$, Z.Thome ${ }^{47}$, A.Tilquin ${ }^{26}$, J.Timmermans ${ }^{30}$, V.G.Timofeev ${ }^{14}$, L.G.Tkatchev ${ }^{14}$, T.Todorov ${ }^{8}$, D.Z.Toet ${ }^{30}$, A.Tomaradze ${ }^{2}$, B.Tome ${ }^{20}$, E.Torassa ${ }^{45}$, L.Tortora ${ }^{40}$, D.Treille ${ }^{7}$, W.Trischuk ${ }^{7}$, G.Tristram ${ }^{6}$, C.Troncon ${ }^{27}, \quad$ A.Tsirou ${ }^{7}$, E.N.Tsyganov ${ }^{14}, \quad$ M-L.Turluer ${ }^{39}$, T.Tuuva ${ }^{13}$ I.A.Tyapkin $^{22}$, M.Tyndel ${ }^{37}$, S.Tzamarias ${ }^{21}$, B.Ueberschaer ${ }^{52}$, S.Ueberschaer ${ }^{52}$, O.Ullaland ${ }^{7}$, V.Uvarov ${ }^{42}$, G.Valenti ${ }^{5}$, E.Vallazza ${ }^{7}$, J.A.Valls Ferrer ${ }^{49}$, C.Vander Velde ${ }^{2}$, G.W.Van Apeldoorn ${ }^{30}$, P.Van Dam ${ }^{30}$, M.Van Der Heijden ${ }^{30}$, W.K.Van Doninck ${ }^{2}$, J.Van Eldik ${ }^{30}$, P.Vaz ${ }^{7}$, G.Vegni ${ }^{27}$, L.Ventura ${ }^{35}$, W.Venus ${ }^{37}$, F.Verbeure $^{2}$, M.Verlato ${ }^{35}$, L.S.Vertogradov ${ }^{14}$, D.Vilanova ${ }^{39}$, P.Vincent ${ }^{24}$, E.Vlasov ${ }^{42}$, A.S.Vodopyanov ${ }^{14}$, M.Vollmer ${ }^{52}$, M.Voutilainen ${ }^{13}$, V.Vrba ${ }^{10}$, H.Wahlen ${ }^{52}$, C.Walck ${ }^{44}$, F.Waldner ${ }^{46}$, A.Wehr ${ }^{52}$, M.Weierstall ${ }^{52}$, P.Weilhammer $^{7}$, A.M.Wetherell ${ }^{7}$, J.H.Wickens ${ }^{2}$, M.Wielers ${ }^{15}$, G.R.Wilkinson ${ }^{34}$, W.S.C.Williams ${ }^{34}$, M.Winter ${ }^{8}$, G.Wormser $^{18}$, K.Woschnagg ${ }^{48}$, A.Zaitsev ${ }^{42}$, A.Zalewska ${ }^{16}$, D.Zavrtanik ${ }^{42}$, E.Zevgolatakos ${ }^{9}$, N.I.Zimin ${ }^{14}$, M.Zito $^{39}$, D.Zontar ${ }^{43}$, R.Zuberi ${ }^{34}$, G.Zumerle ${ }^{35}$

\footnotetext{
${ }^{1}$ Ames Laboratory and Department of Physics, Iowa State University, Ames IA 50011, USA

${ }^{2}$ Physics Department, Univ. Instelling Antwerpen, Universiteitsplein 1, B-2610 Wilrijk, Belgium and IIHE, ULB-VUB, Pleinlaan 2, B-1050 Brussels, Belgium and Faculté des Sciences, Univ. de l'Etat Mons, Av. Maistriau 19, B-7000 Mons, Belgium

${ }^{3}$ Physics Laboratory, University of Athens, Solonos Str. 104, GR-10680 Athens, Greece

${ }^{4}$ Department of Physics, University of Bergen, Allégaten 55, N-5007 Bergen, Norway

${ }^{5}$ Dipartimento di Fisica, Università di Bologna and INFN, Via Irnerio 46, I-40126 Bologna, Italy

${ }^{6}$ Collège de France, Lab. de Physique Corpusculaire, IN2P3-CNRS, F-75231 Paris Cedex 05, France

${ }^{7} \mathrm{CERN}, \mathrm{CH}-1211$ Geneva 23, Switzerland

${ }^{8}$ Centre de Recherche Nucléaire. IN2P3 - CNRS/ULP - BP20, F-67037 Strasbourg Cedex, France

${ }^{9}$ Institute of Nuclear Physics, N.C.S.R. Demokritos, P.O. Box 60228, GR-15310 Athens, Greece

${ }^{10} \mathrm{FZU}$, Inst. of Physics of the C.A.S. High Energy Physics Division, Na Slovance 2, CS-180 40, Praha 8, Czechoslovakia

${ }^{11}$ Dipartimento di Fisica, Università di Genova and INFN, Via Dodecaneso 33, I-16146 Genova, Italy

${ }^{12}$ Institut des Sciences Nucléaires, IN2P3-CNRS. Université de Grenoble 1, F-38026 Grenoble, France

${ }^{13}$ Research Institute for High Energy Physics, SEFT, P.O. Box 9, FIN-00014 University of Helsinki. Finland

${ }^{14}$ Joint Institute for Nuclear Research, Dubna, Head Post Office, P.O. Box 79, 101 000 Moscow, Russian Federation

${ }^{15}$ Institut für Experimentelle Kernphysik, Universität Karlsruhe, Postfach 6980, D-76128 Karlsruhe, Germany

${ }^{16}$ High Energy Physics Laboratory, Institute of Nuclear Physics, Ul. Kawiory 26a, PL-30055 Krakow 30, Poland

${ }^{17}$ Centro Brasileiro de Pesquisas Físicas, rua Xavier Sigaud 150, RJ-22290 Rio de Janeiro, Brazil

${ }^{18}$ Université de Paris-Sud, Lab. de l'Accélérateur Linéaire, IN2P3-CNRS, Bat 200. F-91405 Orsay, France

${ }^{19}$ School of Physics and Materials, University of Lancaster, Lancaster LA1 4YB, UK

${ }^{20}$ LIP, IST, FCUL - Av. Elias Garcia, 14-1 ${ }^{\circ}$, P-1000 Lisboa Codex, Portugal

${ }^{21}$ Department of Physics, University of Liverpool, P.O. Box 147, Liverpool L69 3BX, UK

${ }^{22}$ LPNHE, IN2P3-CNRS, Universités Paris VI et. VII, Tour 33 (RdC), 4 place Jussieu. F-75252 Paris Cedex 05 , France

${ }^{23}$ Department of Physics, University of Lund, Sölvegatan 14, S-22363 Lund, Sweden

${ }^{24}$ Université Claude Bernard de Lyon, IPNL. IN2P3-CNRS, F-69622 Villeurbanne Cedex, France

${ }^{25}$ Universidad Complutense, Avda. Complutense s/n, E-28040 Madrid, Spain

${ }^{26}$ Univ. d'Aix - Marseille II - CPP, IN2P3-CNRS, F-13288 Marseille Cedex 09, France

${ }^{27}$ Dipartimento di Fisica, Università di Milano and INFN, Via Celoria 16, I-20133 Milan, Italy

${ }^{28}$ Niels Bohr Institute, Blegdamsvej 17, DK-2100 Copenhagen 0, Denmark

${ }^{29} \mathrm{NC}$, Nuclear Centre of MFF, Charles University, Areal MFF, V Holesovickach 2, CS-180 00, Praha 8, Czechoslovakia

30 NIKHEF-H, Postbus 41882, NL-1009 DB Amsterdam, The Netherlands

${ }^{31}$ National Technical University, Physics Department, Zografou Campus, GR-15773 Athens, Greece

32 Physics Department, University of Oslo, Blindern, N-1000 Oslo 3, Norway

${ }^{33}$ Dpto. Fisica, Univ. Oviedo, C/P.Jimenez Casas, S/N-33006 Oviedo, Spain

${ }^{34}$ Department of Physics, University of Oxford. Keble Road, Oxford OX1 3RH, UK

${ }^{35}$ Dipartimento di Fisica, Università di Padova and INFN, Via Marzolo 8, I-35131 Padua, Italy

${ }^{36}$ Depto. de Fisica, Pontificia Univ. Católica, C.P. 38071 RJ-22453 Rio de Janeiro, Brazil

${ }^{37}$ Rutherford Applet on Laboratory, Chilton, Didcot OX11 OQX, UK

${ }^{38}$ Dipartimento di Fisica, Università di Roma II and INFN, Tor Vergata, I-00173 Rome, Italy

${ }^{39}$ Centre d'Etude de Saclay, DSM/DAPNIA, F-91191 Gif-sur-Yvette Cedex, France

${ }^{40}$ Istituto Superiore di Sanità, Ist. Naz. di Fisica Nucl. (INFN), Viale Regina Elena 299, I-00161 Rome, Italy

41 C.E.A.F.M., C.S.I.C. - Univ. Cantabria, Avda. los Castros, S/N-39006 Santander, Spain

${ }^{42}$ Inst. for High Energy Physics, Serpukov P.O. Box 35, Protvino, (Moscow Region), Russian Federation

${ }^{43} \mathrm{~J}$. Stefan Institute and Department of Physics, University of Ljubljana, Jamova 39, SI-61000 Ljubljana, Slovenia

${ }^{44}$ Fysikum, Stockholm University, Box 6730, S-113 85 Stockholm, Sweden

${ }^{45}$ Dipartimento di Fisica Sperimentale, Università di Torino and INFN, Via P. Giuria 1, I-10125 Turin, Italy

${ }^{46}$ Dipartimento di Fisica, Università di Trieste and INFN, Via A. Valerio 2, I-34127 Trieste, Italy

and Istituto di Fisica, Università di Udine, I-33100 Udine, Italy

${ }^{47}$ Univ. Federal do Rio de Janeiro, C.P. 68528 Cidade Univ., Ilha do Fundão BR-21945-970 Rio de Janeiro, Brazil

${ }^{48}$ Department of Radiation Sciences, University of Uppsala, P.O. Box 535, S-751 21 Uppsala, Sweden

$49 \mathrm{IFIC}$, Valencia-CSIC, and D.F.A.M.N., U. de Valencia, Avda. Dr. Moliner 50, E-46100 Burjassot (Valencia), Spain

${ }^{50}$ Institut für Hochenergiephysik, Österr. Akad. d. Wissensch., Nikolsdorfergasse 18, A-1050 Vienna, Austria

${ }^{51}$ Inst. Nuclear Studies and University of Warsaw, Ul. Hoza 69, PL-00681 Warsaw, Poland

${ }^{52}$ Fachbereich Physik, University of Wuppertal, Postfach 100127 , D-5600 Wuppertal 1, Germany
} 


\section{Introduction}

Evidence for $\mathrm{B}_{s}^{0}$ meson production has been already obtained at LEP through the partial reconstruction of its semileptonic decay modes [1]. In this paper, the analysis of fully reconstructed decays of the $\mathrm{B}_{s}^{0}$ meson ${ }^{\dagger}$ and the measurement of its mass are presented. The $\mathrm{B}_{s}^{0}-\mathrm{B}_{d}^{0}$ mass difference is expected to be similar to that of the $\mathrm{D}_{s}^{-}-\mathrm{D}^{-}(99.5 \pm 0.6$ $\mathrm{MeV} / \mathrm{c}^{2}$ ) [2]. A value of the $\mathrm{B}_{s}^{0}-\mathrm{B}_{d}^{0}$ mass difference was indirectly inferred from lower energy collider data in an analysis performed by the CUSB collaboration [3]. More recently, the ALEPH Collaboration at LEP and the CDF Collaboration at the Tevatron have published the observation of $\mathrm{B}_{s}^{0}$ decay candidates, obtaining direct measurements of its mass [4].

The decay channels investigated in this analysis have been selected from those leading to all charged final states and with predicted favourable $\mathrm{B}_{s}^{0}$ branching fractions [5]. To reduce the background from reflections of $\mathrm{B}_{d}^{0}$ mesons, only the decay channels for which both the kaons are constrained to an intermediate particle mass have been accepted. After a detailed study on both simulated and real data, three channels were considered : $\mathrm{D}_{s}^{-} \pi^{+}, \mathrm{D}_{s}^{-} \mathrm{a}_{1}^{+}(1260)$ and $\mathrm{J} / \psi \phi$.

The paper is organized as follows: after a brief description of the DELPHI detector components most relevant for the analysis, particle identification and event selection are discussed in section 1 followed by the $\mathrm{B}_{s}^{0}$ reconstruction in section 2 . Section 3 contains then a discussion of the $\mathrm{B}_{s}^{0}$ mass measurement procedure and section 4 the conclusions.

\section{Particle identification and event selection}

A description of the DELPHI apparatus can be found in reference [6]. Only the detectors most relevant for this analysis are described here. The silicon Vertex Detector (VD) provides track reconstruction with high resolution. It consists of three layers of silicon microstrip detectors located at radii $6.5 \mathrm{~cm}, 9 \mathrm{~cm}$ and $11 \mathrm{~cm}$ over a length of 24 $\mathrm{cm}$. The resolution per point in the plane transverse to the beam axis has been measured to be $8.0 \mu \mathrm{m}$.

Muon tagging is based on the associated hits in the Muon Chambers and on the energy deposition in the Hadron Calorimeter.

Hadrons are identified using the specific ionization, $\mathrm{dE} / \mathrm{dx}$, in the Time Projection Chamber (TPC) and the Cherenkov radiation in the barrel Ring Imaging CHerenkov detector (RICH).

The TPC, the principal tracking device of DELPHI, is a cylinder of $30 \mathrm{~cm}$ inner radius, $122 \mathrm{~cm}$ outer radius and a length of $2.7 \mathrm{~m}$. Each end-cap is divided into 6 sector plates each with 192 sense wires used for the particle identification. The energy loss of a charged particle, $\mathrm{dE} / \mathrm{dx}$, is measured by these wires as the truncated mean of the $80 \%$ smallest amplitudes of the wire signals. For particles in hadronic jets, the resolution has been measured to be $7.5 \%$ corresponding to a separation of kaons from pions of 1.5 standard deviations for momenta from $4 \mathrm{GeV} / \mathrm{c}$ to $25 \mathrm{GeV} / \mathrm{c}$. The probabilities for the mass assignment were computed from the measured $\mathrm{dE} / \mathrm{dx}$ and its expected values for the different mass hypotheses $(e, \mu, \pi, K, p)$. An average rejection factor, defined as the ratio between the kaon efficiency and the pion misidentification probability, of about 5 was obtained.

The fiducial volume of the barrel RICH detector [7] covers the polar angular acceptance of $47^{\circ}$ to $133^{\circ}$. This ring imaging Cherenkov detector consists of two volumes in which

\footnotetext{
Throughout the paper the charge-conjugate states are implicitly included.
} 
the Cherenkov photons are produced, one filled with liquid $\mathrm{C}_{6} \mathrm{~F}_{14}$ freon and the other with gaseous $\mathrm{C}_{5} \mathrm{~F}_{12}$ freon. $48 \mathrm{drift}$ tubes containing a photo-sensitive agent (TMAE) are used for the photon detection. The RICH counter separates kaons from pions from 3.5 $\mathrm{GeV} / \mathrm{c}$ up to about $20 \mathrm{GeV} / \mathrm{c}$ using the gas radiator. By adding the information from the liquid radiator, kaon identification is extended down to $1 \mathrm{GeV} / \mathrm{c}$. The probabilities for the mass assignments were computed using the measured Cherenkov angle and the number of detected photons. Kaon candidates were then selected on the basis of the pion and kaon probabilities. A standard kaon selection was defined in order to achieve a high and constant rejection factor. The efficiency of kaon identification was measured using a $D^{*}$ sample (reconstructed through the decay chain $D^{*} \rightarrow D^{0} \pi$ and $D^{0} \rightarrow K \pi$ ) and also by a combined analysis of the RICH data with the $\mathrm{dE} / \mathrm{dx}$ measurement. The misidentification probability was estimated using tagged muon candidates and a very pure pion sample from $\mathrm{K}^{0}$ decays. A mean rejection factor between 12 and 15 was achieved in the momentum interval 3.5 to $20 \mathrm{GeV} / \mathrm{c}$. In order to maximize the efficiency in the $\mathrm{B}_{s}^{0}$ analysis a second, looser kaon selection was introduced giving a rejection factor of about 7 .

Charged particle tracks had to satisfy the following selection criteria: the momentum had to be between 0.4 and $50 \mathrm{GeV} / \mathrm{c}$, the relative error on momentum measurement less than $100 \%$, the projection of their impact parameter relative to the interaction point had to be below $4 \mathrm{~cm}$ in the plane transverse to the beam direction and the distance to the nominal interaction point along the beam direction below $10 \mathrm{~cm}$.

Hadronic events were selected by requiring five or more charged particles and a total energy in charged particles larger than $11 \mathrm{GeV}$ assuming all charged particles to be pions. The hadronic event selection efficiency was estimated from simulation to be $95.0 \pm 0.5 \%$. A total of 750,000 hadronic events was obtained from the 1992 data, of which 450,000 events were taken with the Barrel RICH detector active.

\section{$2 \quad \mathrm{~B}_{s}^{0}$ reconstruction}

The $\mathrm{B}_{s}^{0}$ reconstruction was performed in the $\mathrm{D}_{s}^{-} \pi^{+}, \mathrm{D}_{s}^{-} \mathrm{a}_{1}^{+}(1260)$ and $\mathrm{J} / \psi \phi$ channels. Because of the small number of expected $\mathrm{B}_{s}^{0}$ candidate events, it is needed to keep the combinatorial background and the contamination from $\mathrm{B}_{d}^{0}$ kinematical reflections at a very small level in order to obtain an accurate mass measurement. At this point it is important to notice that all the selected channels lead to a final state formed by two kaons and either two or four lighter particles. The charged $\mathrm{K}$ identification performed by the combined use of the RICH and the $\mathrm{dE} / \mathrm{dx}$ measurements allows clean reconstruction of these states free from the effect of kinematical reflections. The combinatorial background is then further suppressed by cutting on the intermediate state masses and by requiring a well reconstructed secondary decay vertex. Therefore the selection procedure started with the kaon identification, followed by the vertex reconstruction and the kinematic cuts.

At least one kaon identified with the standard RICH selection and having momentum larger than $3.5 \mathrm{GeV} / \mathrm{c}$ was required. For the second kaon, candidates tagged by the loose $\mathrm{RICH}$ selection or the $\mathrm{dE} / \mathrm{dx}$ measurement were also accepted. For channels involving the presence of a $\phi$ resonance, the combinatorial background and kinematical reflections are strongly suppressed by a narrow cut around the the $\phi$ mass. The kaon identification criteria were consequently made looser and only one of the two particles had to be identified as a kaon by either the RICH or the $\mathrm{dE} / \mathrm{dx}$ measurement.

In order to achieve a good accuracy on the position of the secondary vertices and on the track parameters at these vertices, associated VD hits were required for all the 
charged particle tracks. The kaon candidates and additional pions or muons were tested to form common secondary vertices. A minimum vertex probability of $1 \%$ and a positive decay length was required.

Because of the hard fragmentation of the b quark a minimum energy of $25 \mathrm{GeV}$ for the reconstructed $\mathrm{B}$ meson was required. Each particle had in addition to have a momentum above $1 \mathrm{GeV} / \mathrm{c}$, except in the $\mathrm{D}_{s}^{-} \mathrm{a}_{1}(1260)$ channel where this threshold was lowered to $0.5 \mathrm{GeV} / \mathrm{c}$ for the least energetic track, in order to keep a good efficiency. Kaon pairs within $\pm 10 \mathrm{MeV} / \mathrm{c}^{2}$ of the $\phi$ mass and $\mathrm{K} \pi$ pairs within $\pm 80 \mathrm{MeV} / \mathrm{c}^{2}$ of the $\mathrm{K}^{*}(892)^{0}$ mass were taken as decay product of the resonance. These cuts correspond to \pm 2.5 and \pm 2.0 times the observed Gaussian widths for the $\phi$ and the $\mathrm{K}^{*}(892)^{0}$ respectively. Candidate $\mathrm{B}_{s}^{0}$ mesons were accepted in a $300 \mathrm{MeV} / \mathrm{c}^{2}$ mass interval above the $\mathrm{B}_{d}^{0}$ mass, i.e. in the range from $5.28 \mathrm{GeV} / \mathrm{c}^{2}$ to $5.58 \mathrm{GeV} / \mathrm{c}^{2}$. The combined reconstruction efficiencies for the different channels were measured using Monte Carlo generated events including full detector simulation. They were found to be in the range from $15 \%$ to $20 \%$.

\subsection{The $\mathbf{D}_{s}^{-} \pi^{+}$and $\mathbf{D}_{s}^{-} \mathbf{a}_{1}^{+}(1260)$ channel}

After applying the cuts described above, a $\mathrm{D}_{s}^{-}$meson was reconstructed in the two modes $\phi \pi$ or $\mathrm{K}^{*}(892)^{0} \mathrm{~K}$. The two distinct secondary vertices of the $\mathrm{B}_{s}^{0}$ decay chain were reconstructed in the following way. $\mathrm{A} \mathrm{D}_{s}^{-}$vertex was formed with the association of the kaons and a pion satisfying the $\phi$ or the $\mathrm{K}^{*}(892)^{0}$ intermediate mass constraint. The $\mathrm{KK} \pi$ combination was then accepted as a $\mathrm{D}_{s}^{-}$candidate if its momentum was larger than $4 \mathrm{GeV} / \mathrm{c}$ and its mass between 1.93 and $2.01 \mathrm{GeV} / \mathrm{c}^{2}$, corresponding to \pm 2.0 times its observed Gaussian width. The $\mathrm{D}_{s}^{-}$candidate was then extrapolated backwards to form a second vertex with the remaining pions. The primary vertex was obtained by the intersection of the beam spot with the extrapolated $\mathrm{B}_{s}^{0}$ track. The decay distance between the $\mathrm{D}_{s}^{-}$vertex and the primary vertex and between the $\mathrm{B}_{s}^{0}$ vertex and the primary vertex had to be larger than twice the associated error.

For the $\mathrm{D}_{s}^{-} \mathrm{a}_{1}^{+}(1260)$ mode, the three pion system had to be compatible with a $\mathrm{a}_{1}(1260)$ decay within $\pm 250 \mathrm{MeV} / \mathrm{c}^{2}$ of its mass. Because of the larger combinatorial background predicted by simulation, only the $\phi \pi$ mode was used to reconstruct $\mathrm{D}_{s}^{-}$mesons in this channel. One event was found in each of the two channels. The display of the $\mathrm{D}_{s}^{-} \mathrm{a}_{1}^{+}(1260)$ candidate is shown in figure 1 .

\section{$2.2 \quad$ The $\mathbf{J} / \psi \phi$ channel}

$\mathrm{J} / \psi$ mesons have been reconstructed through their decay into muon pairs. Opposite sign muons with an invariant mass within $100 \mathrm{MeV} / \mathrm{c}^{2}$ of the nominal $\mathrm{J} / \psi$ mass and with an energy larger than $10 \mathrm{GeV}$ were taken as $\mathrm{J} / \psi$ candidates. In this decay channel only one secondary vertex is present. This was reconstructed by requiring the four charged

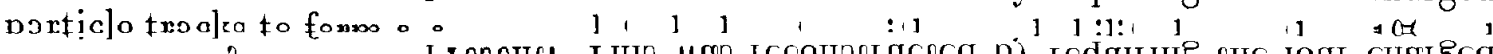

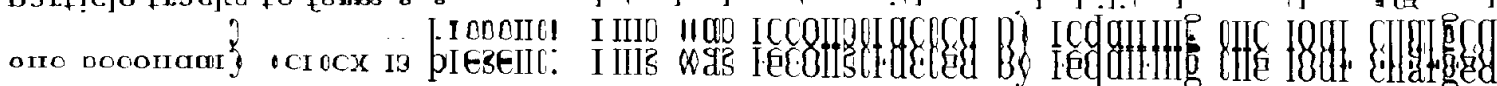
particle tracks to form a common detached vertex with a probability larger than $1 \%$ and a decay distance from the $Z^{0}$ production meint larger than twice its associated error The

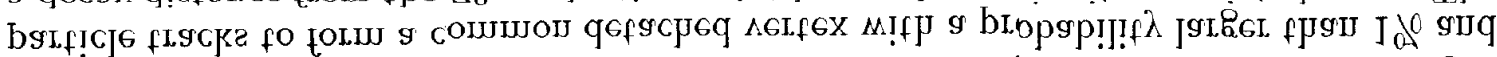

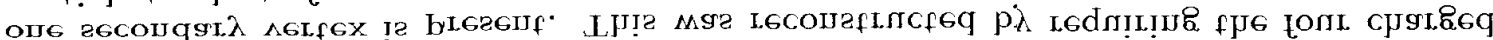

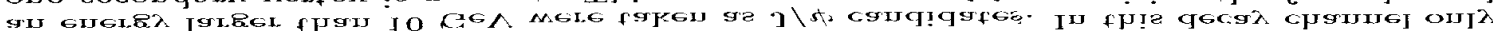

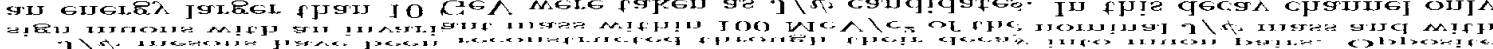

$\mathrm{J} / \psi$ mesons have been reconstructed through their decay into muon pairs. Opposite sign muons with an invariant mass within $100 \mathrm{MeV} / \mathrm{c}^{2}$ of the nominal $\mathrm{J} / \psi$ mass and with an energy larger than $10 \mathrm{GeV}$ were taken as $\mathrm{J} / \psi$ candidates. In this decay channel only one secondary vertex is present. This was reconstructed by requiring the four charged particle tracks to form a common detached vertex with a probability larger than $1 \%$ and a decav distance from the $7^{0}$ nrndurtion noint lomman than twimn : 


\section{$3 \quad \mathbf{B}_{s}^{0}$ mass measurement}

The invariant mass distribution for the three decay channels considered in this analysis is shown in Figure 2. Three $\mathrm{B}_{s}^{0}$ decay candidates have been reconstructed. In order to determine for each candidate the most precise mass, a global constrained fit was performed on the three candidates forcing all the intermediate states $\left(\mathrm{D}_{s}^{-}, \mathrm{J} / \psi, \phi\right)$ to their known masses and recomputing the secondary vertices. Furthermore, all remaining tracks in the $\mathrm{B}_{s}^{0}$ hemisphere with the addition of the reconstructed $\mathrm{B}_{s}^{0}$ track and the beamspot constraint were used to form the primary vertex. A global fit probability larger than $1 \%$ was required. All the three candidates fulfilled this cut. In the simulated data, the $\chi^{2}$ probability distribution was found to be flat for $\mathrm{B}_{s}^{0}$ events. The difference between the observed and generated $\mathrm{B}_{s}^{0}$ mass divided by its computed mass error was found to be well described by a normal distribution. The mass errors of the geometrical vertex fit were checked using a sample of $\mathrm{J} / \psi \rightarrow \mu \mu$ candidates. Again the computed errors agreed with the fitted mass resolution.

The relevant properties of each of these $\mathrm{B}_{s}^{0}$ candidates are listed in Table 1: the reconstructed mass, the closer to the $\mathrm{B}_{d}^{0}$ mass ${ }^{\ddagger}$ of the two possible masses obtained when changing a kaon into a pion, the $\mathrm{B}_{s}^{0}$ energy and decay length.

Table 1: Properties of the three $\mathrm{B}_{s}^{0}$ events.

\begin{tabular}{|c|c|c|c|c|c|}
\hline & mode & $\begin{array}{c}\mathrm{B}_{s}^{0} \text { mass } \\
\left(\mathrm{MeV} / \mathrm{c}^{2}\right)\end{array}$ & $\begin{array}{c}\text { Reflected mass } \\
\left(\mathrm{MeV} / \mathrm{c}^{2}\right)\end{array}$ & $\begin{array}{c}\text { Energy } \\
(\mathrm{GeV})\end{array}$ & $\begin{array}{c}\text { Decay } \\
\text { length }(\mathrm{mm})\end{array}$ \\
\hline 1 & $\mathrm{D}_{s}^{-}(\phi \pi) \pi$ & $5325 \pm 32$ & 5200 & 27.1 & 2.3 \\
2 & $\mathrm{D}_{s}^{-}(\phi \pi) \mathrm{a}_{1}$ & $5345 \pm 32$ & 5130 & 26.7 & 1.4 \\
3 & $J / \psi \phi$ & $5389 \pm 16$ & 5275 & 36.9 & 1.6 \\
\hline
\end{tabular}

The weighted average of the masses of the three candidates leads to the result: $\mathrm{m}_{\mathrm{B}_{s}}=(5371 \pm 13$ (stat) $) \mathrm{MeV} / \mathrm{c}^{2}$. The corresponding $\chi^{2}$ is 4 for 2 degrees of freedom and the observed mass r.m.s. is $32 \mathrm{MeV} / \mathrm{c}^{2}$ in agreement with the expected resolution.

Several sources of error for the $\mathrm{B}_{s}^{0}$ mass measurement have been studied.

Firstly it is important to make sure that the observed $\mathrm{B}_{s}^{0}$ candidates are not due to reflection of the more abundantly produced $\mathrm{B}_{d}^{0}$ meson when a misidentified pion is attributed the kaon mass. The expected number of $\mathrm{B}_{d}^{0}$ reflections in the $\mathrm{D}_{s}^{-}$decay channels has been computed taking into account the different $\mathrm{B}_{d}^{0}$ and $\mathrm{B}_{s}^{0}$ production rate, the pion rejection factor achieved with the RICH and the intermediate mass constraint. The result is 0.10 event. In fact neither of the two candidates in the $\mathrm{D}_{s}^{-}$channels is compatible with the $\mathrm{B}_{d}^{0}$ mass on assigning the pion mass to either of the kaon candidates. For the $\mathrm{J} / \psi \phi$ candidate, one kaon is identified by the specific ionization and its reflected mass is compatible with the $\mathrm{B}_{d}^{0}$ hypothesis. A possible reflection from the non resonant $B_{d}^{0} \rightarrow \mathrm{J} / \psi \mathrm{K} \pi$ decay has been studied. The reflected mass spectrum for the $\mathrm{K} \pi$ system, imposing a kaon mass to the pion, is a broad structure centered on $1.6 \mathrm{GeV} / \mathrm{c}^{2}$ so that the mass range used for the $\phi$ resonance contains only $0.6 \%$ of the generated sample. The global probability that this event comes from a $\mathrm{B}_{d}^{0}$ meson decay is $10 \%$ and contributes 0.10 events to the reflection background. As a further check, the $\mathrm{B}_{s}^{0}$ search was performed on two simulated data samples: the first one corresponding to 1.5 million hadronic $\mathrm{Z}^{0}$ decays and the second one being an enriched sample of $B$ particles decaying in all charged final states.

\footnotetext{
†Particle Data Group world average value [8] $(5278.7 \pm 2.0) \mathrm{MeV} / \mathrm{c}^{2}$
} 
Only genuine $\mathrm{B}_{s}^{0}$ decays were selected by the cuts used in the analysis. Considering the three decay modes taken into account in this analysis, the expected background from $\mathrm{B}_{d}^{0}$ reflections has been evaluated to be 0.20 events.

The combinatorial background represents a second source of errors. No events other than the $\mathrm{B}_{s}^{0}$ candidates were found in the mass region 4.5 to $6.0 \mathrm{GeV} / \mathrm{c}^{2}$. The combinatorial background was evaluated from the real data by scaling the number of events in this mass region before and after the kaon identification and the vertex cuts. The expected total number of background events in the $\mathrm{B}_{s}^{0}$ candidate sample is $(0.05 \pm 0.04) /\left(50 \mathrm{MeV} / \mathrm{c}^{2}\right)$, consistent with the observation of no event outside the signal region.

A third source of bias comes from the incomplete reconstruction of decays through a $\mathrm{D}_{s}^{*}$. Rates for the $\mathrm{B}_{s}^{0}$ to $\mathrm{D}_{s}^{*}$ decays are expected to be comparable with those for the direct decay to $\mathrm{D}_{s}^{-}$[5]. Since the photons produced in these decays are not included in this analysis, they contribute a broad structure beside the genuine $\mathrm{B}_{s}^{0}$ mass peak.

In order to take into account the possible distortions of the measured mass and the contribution to the error from these sources, a global likelihood fit was performed. For each event, the probability density function includes the signal and, in the $\mathrm{D}_{s}^{-}$channels, the contribution from incomplete $\mathrm{D}_{s}^{*}$ reconstruction. The signal is described by a Gaussian with its centre and width corresponding to the measured mass and error. The shape for the decays with a $\mathrm{D}_{s}^{*}$ was obtained from a mass constrained fit of the event, allowing a free photon and imposing the $\mathrm{D}_{s}^{*}$ mass. The weights of the genuine $\mathrm{D}_{s}^{-}$contribution and of that from the $\mathrm{D}_{s}^{*}$ were set equal (i.e. assuming an equal production of $\mathrm{D}_{s}^{-}$and $\mathrm{D}_{s}^{*}$ ). The mass distributions of the background were assumed to be flat in all channels, while the distribution of the $\mathrm{J} / \psi K \pi$ reflection was derived from Monte Carlo simulation in which the compatibility of the " $K K$ " mass (when attributing the $K$ mass to the $\pi$ ) with the $\phi$ mass was imposed.

The free parameters of the fit are the $\mathrm{B}_{s}^{0}$ mass and the expected number of signal events in each of the three channels. The resulting likelihood distribution is shown in fig. $3 \mathrm{~b}$ as a function of the $\mathrm{B}_{s}^{0}$ mass.

This fit leads to the value of $\mathrm{m}_{\mathrm{B}_{\mathrm{s}}}=(5374 \pm 16) \mathrm{MeV} / \mathrm{c}^{2}$ where the error accounts for the statistical error and the systematical error from the background, the kinematical reflections and the $\mathrm{D}_{s}^{*}$ channels. The stability of the result when changing the ratio of $\mathrm{D}_{s}^{-} / \mathrm{D}_{s}^{*}$ production or the reflection probability for the $\mathrm{J} / \psi \phi$ event was also checked: a change of these values by $50 \%$ does not vary the value of the fitted mass.

An additional source of systematic error comes from the absolute mass scale calibration. Charmed and beauty mesons were used to check this calibration. The reconstructed masses of $\mathrm{J} / \psi$ and $\mathrm{D}_{s}^{-}$particles were found to be in good agreement with the Particle Data Group values [8]. High momentum $\mathrm{D}^{0}$ decaying into $\mathrm{K}^{-} \pi^{+} \pi^{+} \pi^{-}$and $\mathrm{D}^{-}$decaying into $\mathrm{K}^{+} \pi^{-} \pi^{-}$were used for evaluating the momentum scale uncertainty. From their measured masses of $1866 \pm 3 \mathrm{MeV} / \mathrm{c}^{2}$ and $1868 \pm 2 \mathrm{MeV} / \mathrm{c}^{2}$ respectively this uncertainty has been estimated to be $0.11 \%$. The comparison of the masses measured for the $B^{0}$ and $B^{-}$ meson candidates in channels analogous to those used in this analysis with Particle Data Group value confirmed this result. The use of the mass constrained fit further limits the possible distortions originating from the absolute mass scale. The contribution from this systematic error source was estimated to be $2 \mathrm{MeV} / \mathrm{c}^{2}$. The final result is thus : $\mathrm{m}_{\mathrm{B}_{\mathrm{s}}}=(5374 \pm 16 \pm 2) \mathrm{MeV} / \mathrm{c}^{2}$. 


\section{Conclusions}

$\mathrm{B}_{s}^{0}$ mesons have been detected through fully reconstructed final states. From three candidates the $\mathrm{B}_{s}^{0}$ meson mass has been measured to be $(5374 \pm 16 \pm 2) \mathrm{MeV} / \mathrm{c}^{2}$, where the first error represents the statistical error and the systematic error from the background sources combined and the second error represents the mass scale uncertainty. This gives a mass difference between the $\mathrm{B}_{s}^{0}$ and $\mathrm{B}_{d}^{0}$ meson of $(95 \pm 16) \mathrm{MeV} / \mathrm{c}^{2}$ using the world average value of the $\mathrm{B}_{d}^{0}$ meson mass. This mass difference is in good agreement with the existing results and the predictions of the quark model.

\section{Acknowledgements}

We are greatly indebted to our technical collaborators and to the funding agencies for their support in building and operating the DELPHI detector, and to the members of the CERN-SL Division for the excellent performance of the LEP collider. 


\section{References}

[1] P.Abreu et al., DELPHI collab., Phys. Lett. B289 (1992) 199;

D. Buskulic et al., ALEPH collab., Phys. Lett. B294 (1992) 145;

P. D. Acton et al., OPAL collab., Phys. Lett. B295 (1992) 357.

[2] U. Aglietti, Phys. Lett. B281 (1992) 341;

T. Ito, T. Morii, M. Tanimoto, Z. Phys. C59 (1993) 57;

Z. Guralnik and A. V. Manohar, Phys. Lett. B302 (1993) 103.

[3] J. Lee-Franzini et al., CUSB collab., Phys. Rev. Lett. 65 (1990) 2947.

[4] D. Buskulic et al., ALEPH collab., Phys. Lett. 311 (1993) 425;

F. Abe et al., CDF collab., Phys. Rev. Lett. 71 (1993) 1685.

[5] J. Bijnens and F. Hoogeveen, Phys. Lett. B283 (1992) 434.

[6] DELPHI collab., Nucl. Instr. and Meth. A303 (1991) 233.

[7] E. G. Anassontzis et al., Nucl. Instr. and Meth. A323 (1992) 351.

[8] Particle Data Group, K.Hikasa et al., Phys. Rev. D45 (1992). 


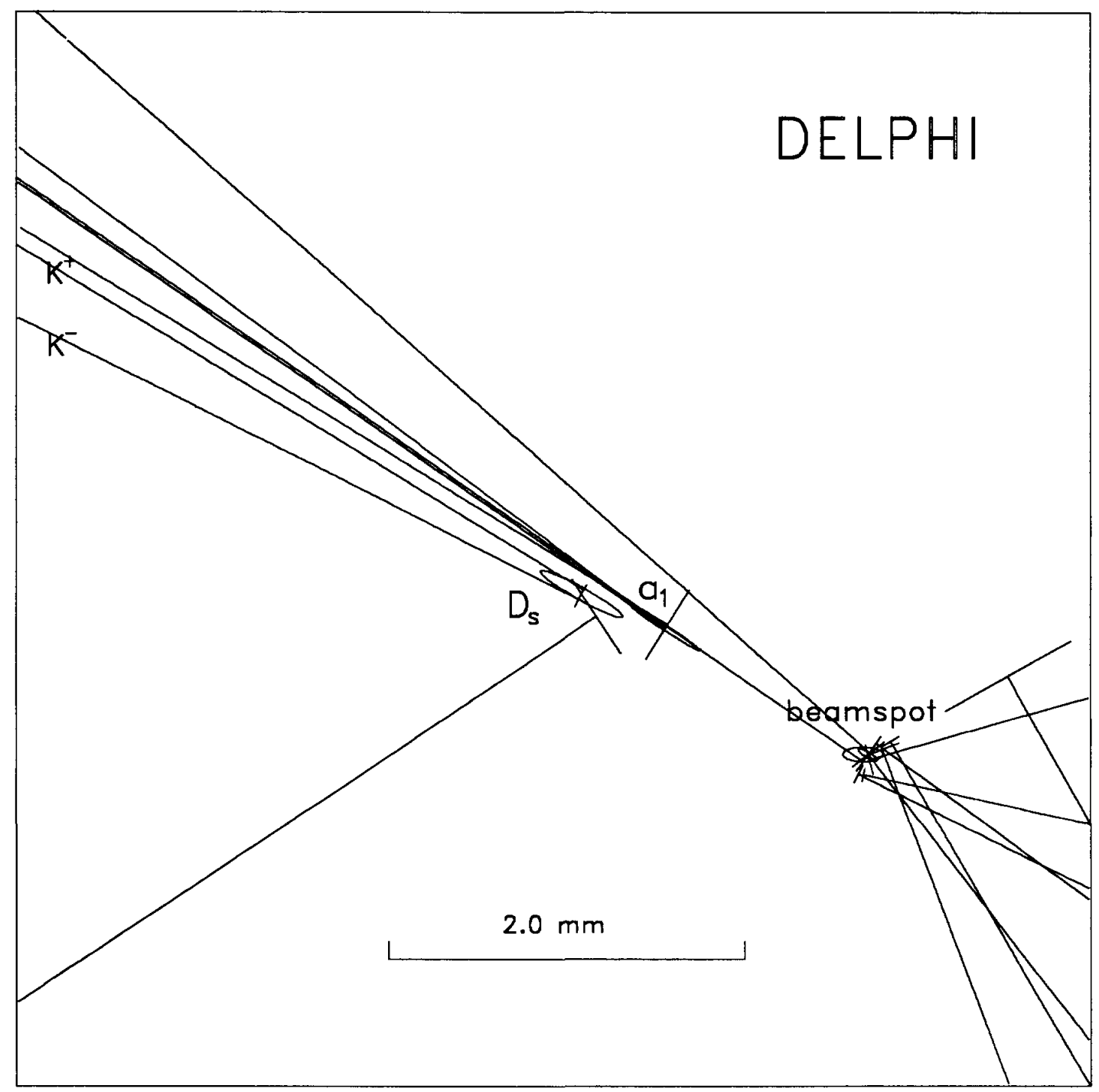

Figure 1: Display of the $r-\phi$ projection of the reconstructed decay chain for the $\mathrm{D}_{s}^{-} \mathrm{a}_{1}^{+}(1260)$ candidate. The ellipses correspond to the $1 \sigma$ region around the reconstructed vertices and the bars on the tracks represent the computed extrapolation errors. 


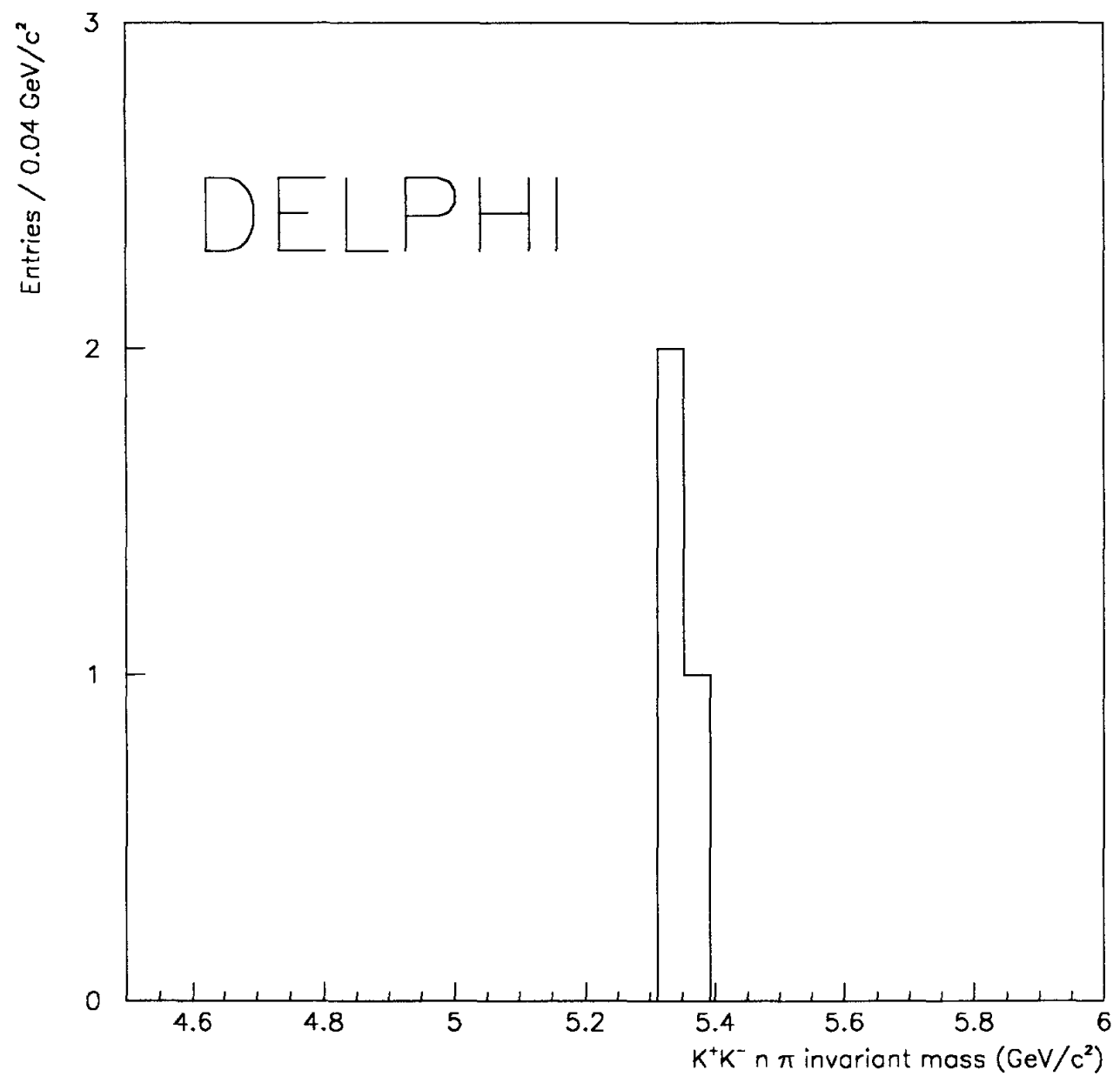

Figure 2: The invariant mass distribution for the $\mathrm{D}_{s}^{-} \pi^{+}, \mathrm{D}_{s}^{-} \mathrm{a}_{1}^{+}(1260)$ and $\mathrm{J} / \psi \phi$ decay channels. No entry outside the $\mathrm{B}_{s}^{0}$ mass acceptance interval from 5.28 to $5.58 \mathrm{GeV} / \mathrm{c}^{2}$ is found. 

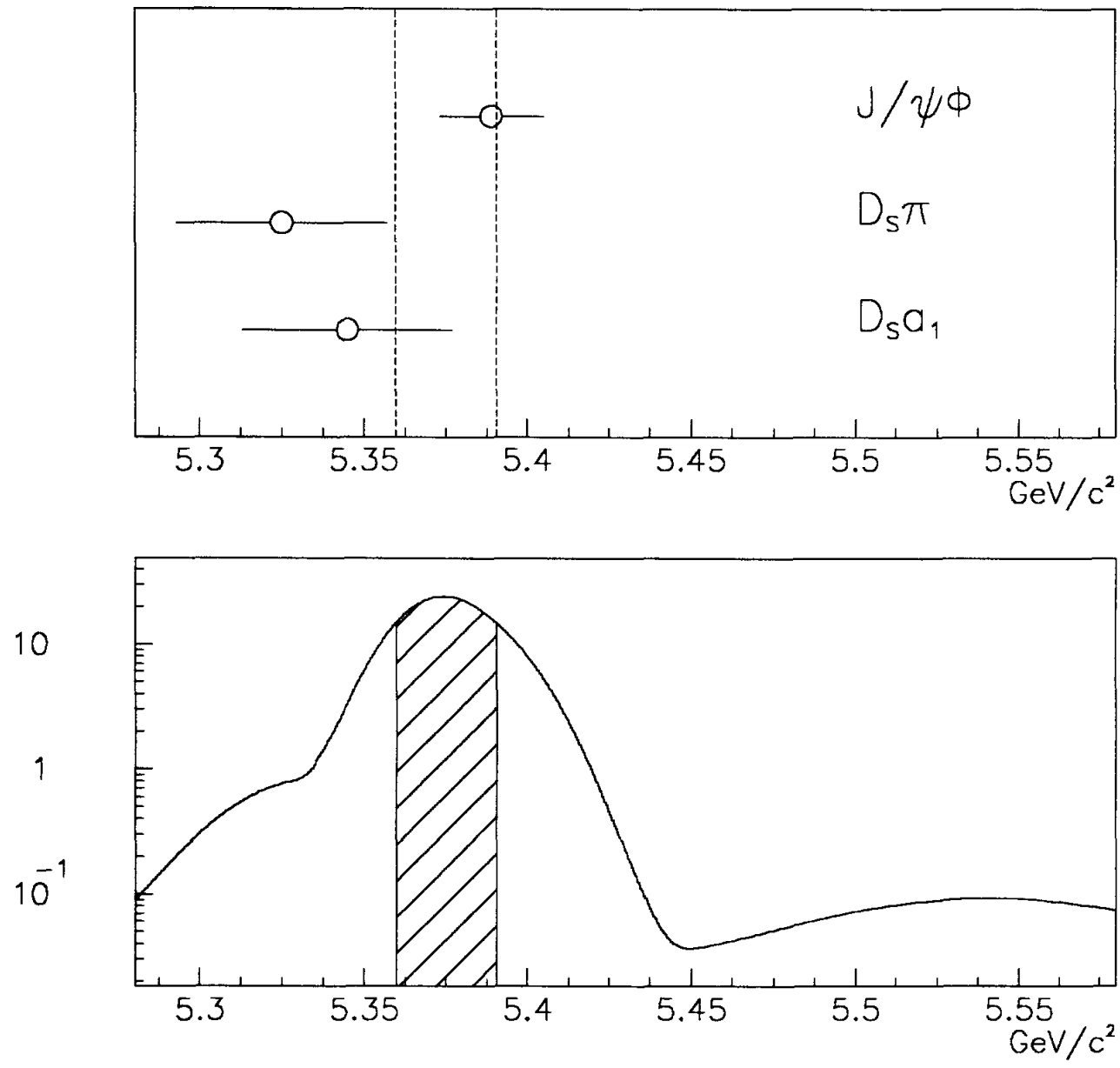

Figure 3: (a) The three $\mathrm{B}_{s}^{0}$ candidate masses and errors after the global constrained fit. (b) The likelihood distribution obtained from the fit with the resulting $\mathrm{B}_{s}^{0}$ mass and the $68 \%$ confidence interval. The small bump at the right corresponds the contribution from the incompletely reconstructed decays with a $\mathrm{D}_{s}^{*}$. 
